\title{
Design and Implementation of a Lightweight Lower Extremity Exoskeleton
}

\author{
Jiamin $\mathrm{Mu}^{1}$, Hongzhou Jiang ${ }^{2}$, Yuxiang Hua ${ }^{1}$, Jie Zhao ${ }^{1}$ and Yanhe Zhu ${ }^{1, a}$ \\ ${ }^{1}$ State Key Laboratory of Robotics and System, Harbin Institute of Technology, Harbin 150001, China \\ ${ }^{2}$ Department of Fluid Control and Automation, Harbin Institute of Technology, Harbin 150001, China
}

\begin{abstract}
This paper summarizes the biomechanics simulation and design of a lightweight lower extremity exoskeleton. The biomechanics simulation was carried out using LifeMOD to obtain joint torques of human body in the condition of its applications including walking on flat ground, ascending stairs, sitting down and standing up which provided the design criteria of actuating torques. The anthropomorphically based exoskeleton has 7 degrees of freedom per leg, two of which are powered by brushless DC motors, and the total mass is less than $12 \mathrm{~kg}$ due to the use of carbon fiber material and the compact structural design and optimization. Controlling and sensing system based on CANopen communication protocol is proposed which can provide high communication efficiency and reliability for the control of the exoskeleton.
\end{abstract}

\section{Introduction}

Nowadays, exoskeletons have been widely used in medical field. Possible applications include helping wearers move and recover from the dysfunction of lower limbs. Compared with manual wheelchair propulsion for locomotion, powered exoskeletons can adapt to a wide range of terrains and load the legs which is a key factor in maintaining bone strength [1]. Due to these advantages, the Rewalk [2] was developed for individuals with spinal cord injury (SCI) to walk independently. The system is self-contained and subject-directed. Users control their own walking through activating the locomotion mode programs and minor trunk movements. Meanwhile, another exoskeleton, Ekso [3], had been developed at Ekso Bionics. Differently, it was designed for muscle and nervous system rehabilitation combining with walking sticks under the help of physical therapist. After later improvement, motion sensors that communicate with the legs of the exoskeleton are mounted to the walking sticks, allowing the wearer to take complete control. Before the above two exoskeletons, Yoshiyuki Sankai from the University of Tsukuba introduced a series of lightweight power assist devices, HAL [4, 5]. Different from previous exoskeletons, HAL is driven by predicting motion intention of the human based on sEMG. In addition, still in development are several other lower extremity exoskeletons designed to aid disabled people $[6,7]$.

Interiorly, the most representative researches include the rehabilitative lower limb exoskeleton of SIAT [8], and the auxiliary device of UESTC [9]. At present, these two exoskeletons have entered the stage of small-scale clinical testing. Shanghai Fourier Intelligent Technology

\footnotetext{
a Corresponding author: yhzhu@hit.edu.cn
}

Corporation developed the first commercial lower limb exoskeleton, Fourier X1, for rehabilitation.

In this paper, we designed this exoskeleton which is shown in Fig. 1. It comprises two powered anthropomorphic legs, a power supply and a set of controlling and sensing system. The exoskeleton has hip, knee and ankle joints, like in a person, while the details of these joints differ from human joints. The ranges of these joints are considered to cover normal range of human motion while avoiding overextension. All sensors are embedded into the exoskeleton to improve the convenience of wearing.

This paper is organized as follows. In section 1, the research status of exoskeletons in the field of medical rehabilitation at home and abroad is introduced. Then the biomechanics simulation and analysis of human body are carried out to obtain the design criteria of actuating torques of the exoskeleton in section 2. The mechanical design architecture and controlling and sensing system are illustrated in section 3 and 4, respectively. Finally, some conclusions are drawn in the last section.

\section{Biomechanics simulation of human lower limbs}

Anthropomorphic method is adopted in the design of the exoskeleton with limb masses and inertias same as in a human. The required joint torques for the exoskeleton to perform a given motion are approximated as that required by a similarly sized human performing the same motion. Therefore, the ratio of joint torque to the mass of human body in the process of specified motion is used to determine the design criteria of actuating torques. For this purpose, biomechanics simulation of human body is introduced based on three kinds of motions including 

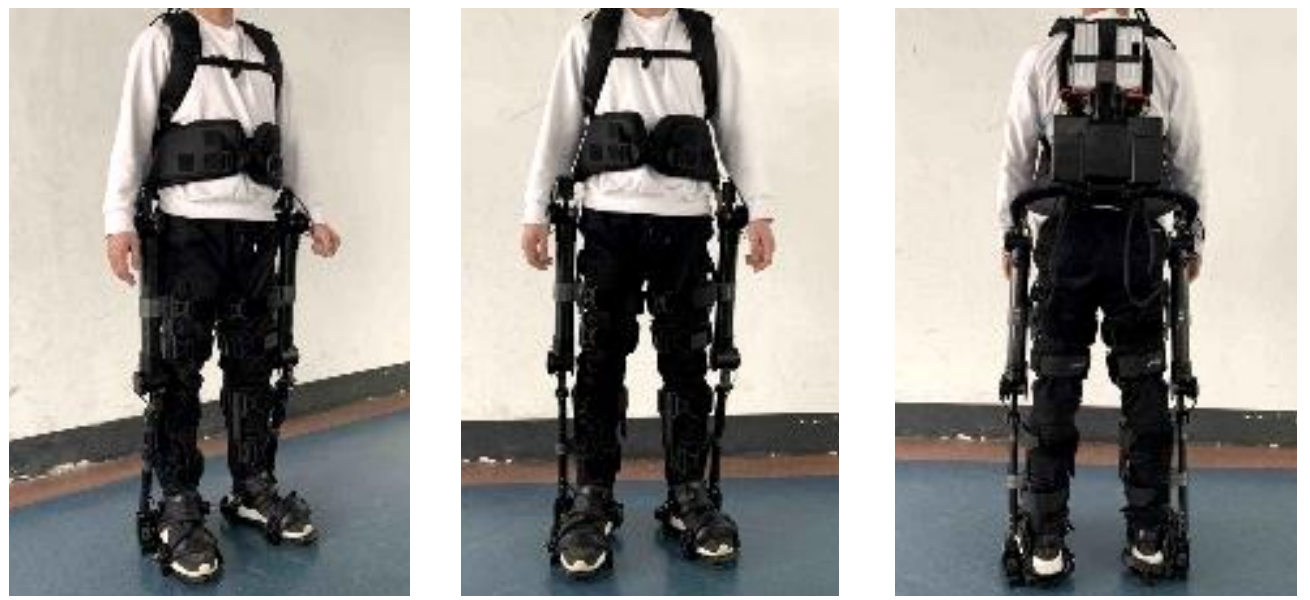

Figure 1. Electricity-powered, 14-DOF, lower limb exoskeleton walking on flat ground, climbing stairs and sitting down and standing up which are common in daily life.

In the study, LifeModeler modeling system is employed for the simulation. The musculoskeletal model of human body used in the biomechanics simulation for the three motions mentioned earlier is shown in Fig. 2. The musculoskeletal model includes skeletons, joints and muscles, and their shapes, masses and inertias are derived from anthropometric databases. The yellow spheres are motion agents, the positions of which are determined by motion capture data, and the red spheres are feature points attached to the model. They are connected by bushing elements. Each joint's degree of freedom contains a moment function, which is similar to the spring damping model. Correspondingly, learning and contraction units are contained in muscles. They are trained via inverse dynamics simulation and then generate torques or forces to cause the model to move in the manner as developed from the inverse dynamics simulation.

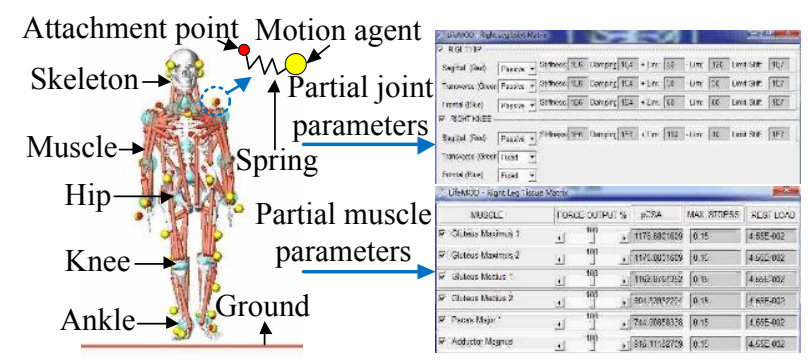

Figure 2. Musculoskeletal model used in the biomechanics simulation

Fig. 3 shows the process of forward dynamics simulation in the condition of the three kinds of motions mentioned earlier. The musculoskeletal model is propelled by joint torques to maintain balance and achieve position control for each part of the model while motion agents are frozen. Contact constraint is applied between the human body model and the environment, generating reaction force and friction to support the model and provide the power for the movement. During this process, joint torques are recorded.

The curves of joint torques obtained from forward biomechanics simulation are shown in Fig. 4. During one cycle of walking on flat ground, toe-off occurs at $40 \%$ of the step cycle and heel strike occurs at $80 \%$ of the step cycle. The hip torque is relatively symmetric; hence, a bidirectional hip actuator is required. Positive extension torque is required in early stance as the hip supports the load on the stance leg. It is negative in late stance and early swing as the hip propels the leg forward during swing. In late swing, the torque goes positive as the hip decelerates the leg prior to heel strike. As for peak moment, that required by hip is larger, which is numerically about 0.7 times of the body weight. In the process of ascending stairs, toe-off of right leg occurs at $30 \%$ of the step cycle, erenow, left knee contributes the most to support the human body. Heel strike of right leg occurs at $52 \%$ of the step cycle, after that, right knee lifts the human body upward. The required moment is about 1.1 times of the body weight. Knee joints play a major role in sitting down and standing up, and the maximum torque is about 1.15 times of the body weight. Relative researches indicate that the muscle strength of the elderly is 18 to 20 percent lower than that of the middle-aged, which are the required torques the exoskeleton acts on the wearer.

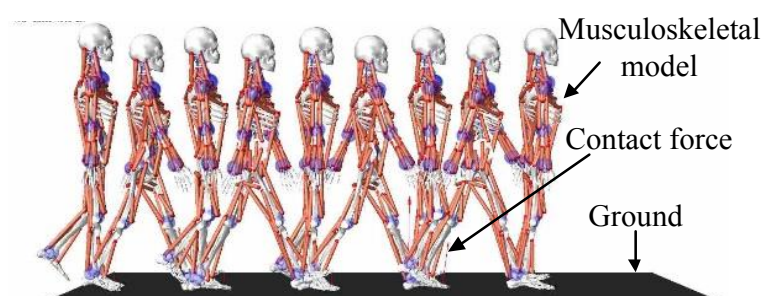

(a) Walking on flat ground
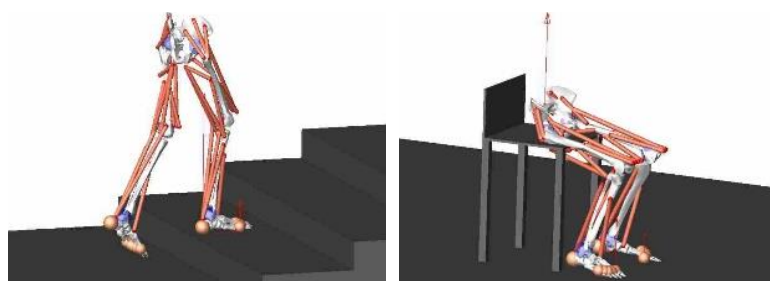

(b) Ascending stairs

(c) Sitting down and standing up

Figure 3. The forward dynamics process of biomechanics simulation

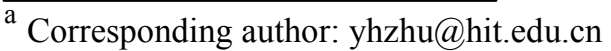




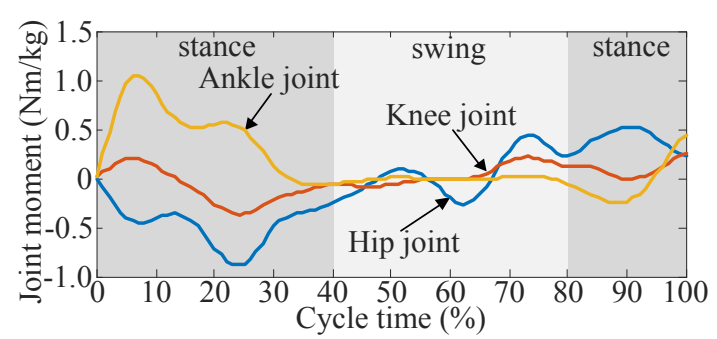

(a) Walking on flat ground

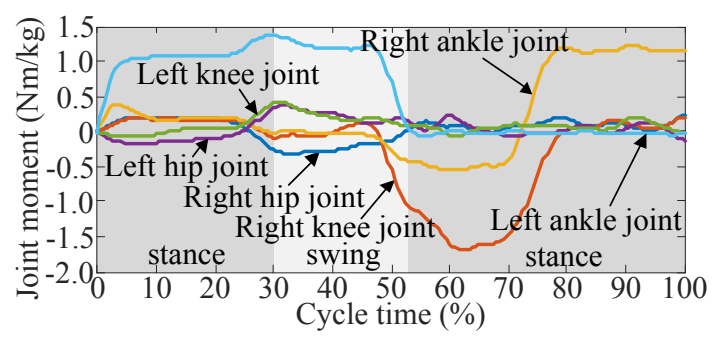

(b) Ascending stairs

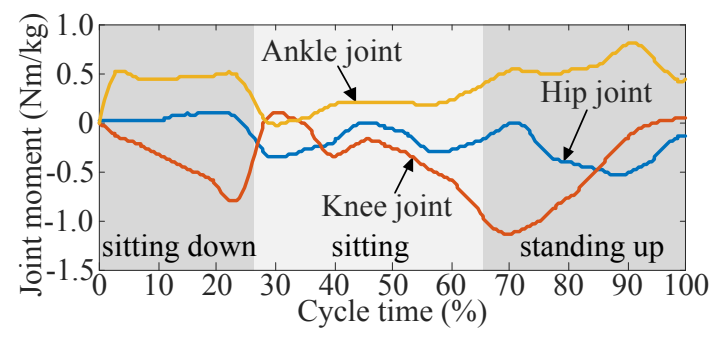

(c) Sitting down and standing up

Figure 4. Joint flexion/extension torque measured from biomechanics simulation

\section{Design and optimization of structure}

\subsection{Mechanical design and construction}

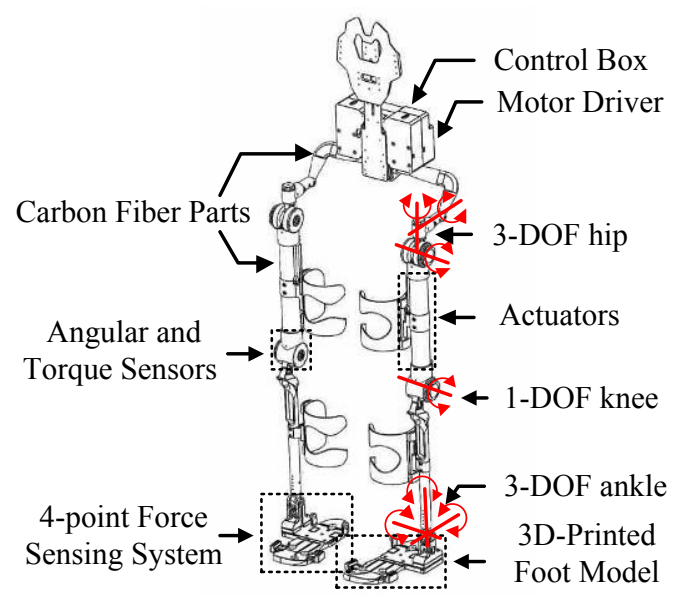

Figure 5. Design architecture of the exoskeleton

The main components of the lightweight exoskeleton are shown in Fig. 5. The exoskeleton has 14 degrees of freedom (DOF), actuating all of them creates unnecessarily high-power consumption and structural complexity. Instead, the active DOF of hip and knee joints are arranged on the sagittal plane in order to support the wearer in the direction of gravity. Therefore, each leg of the exoskeleton has a 3-DOF hip with active DOF in sagittal plane and passive DOF in the other two directions, a 1-DOF knee for flexion/extension movement, and an ankle with three passive DOF.

As shown in Fig. 5, the torso connects to the hip structure. The power supply, controller, motor drivers and a inclinometer mount to the rear side of the torso. The inclinometer gives the absolute angle reference for the control algorithm. A set of harness mounts to the front of the torso to hold the exoskeleton to the wearer. Waist structure made of carbon fiber is customized to reduce the weight.

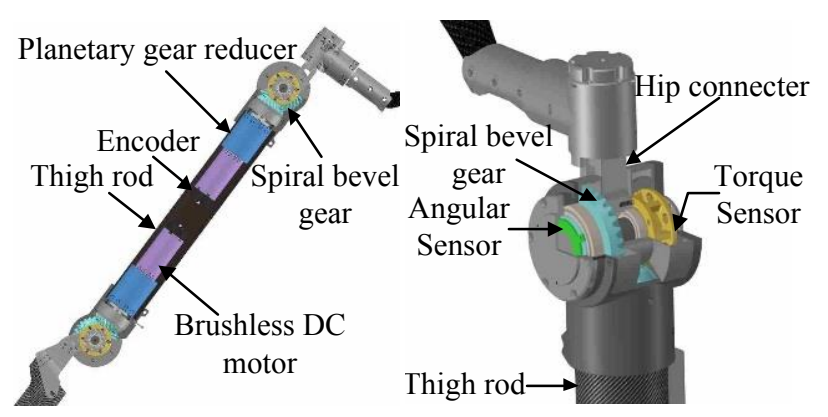

(a) Inner structure of thigh rod

(b) Structure of hip joint

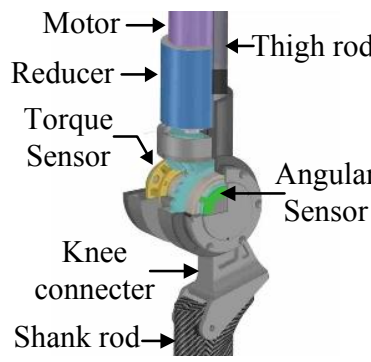

(c) Structure of knee joint

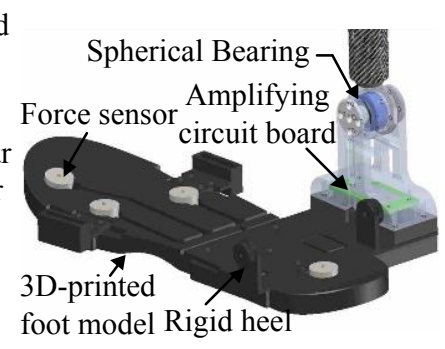

(d) Structure of ankle joint
Figure 6. Detailed mechanical structure of the exoskeleton

The main function of the exoskeleton's shanks and thighs are to provide structural support and to connect the flexion/extension joints together. To reduce the weight of the exoskeleton, the thighs and shanks are customized using carbon fiber. Several bindings mount to them to connect exoskeleton to the legs of operator. As shown in Fig. 6(a), inside the thigh which is designed as a cylinder to suit the shape of motor and reducer for structural compactness purpose, two actuators are arranged symmetrically to generate torques for hip and knee joints. Each actuator comprises a brushless DC motor, an encoder, a planetary gear reducer and a pair of spiral bevel gears. Using of the spiral bevel gears simplifies the dynamic center position of the thigh inertia and having better performance in responding to the quick action of the wearer and absorbing the shock. The reduction ratio of the planetary gear reducer and the spiral bevel gears is 156:1 and 1.5:1 respectively, accordingly, it can provide the rated torque of $22.3 \mathrm{Nm}$ for hip and knee joints. According to the analysis in section 2, the joint torques can meet the requirement of assisting.

Between torso and legs, there are two passive DOF for hip abduction/adduction and rotation to meet the requirements of human motions. The structure of hip joint is similar to that of knee joint, as shown in Fig. 6(b) 
and (c). The hip connecter is connected with the hip axis supporting by a pair of bearings. Meanwhile, a torque sensor and a spiral bevel gear are supported by the axis. The hip torque is transferred via keys between the gear, axis and torque sensor and screw connections between the torque sensor and hip connecter. The proposed arrangement can reduce the influence of radial force on the stress distribution of the sensor. The torque sensor is designed as a link of transmission to detect the torque at hip joint. Compared with the force sensor installed between human and exoskeleton, the torque sensor's detection result is not affected by wearing and more reliable.

Mechanical structure of ankle is shown in Fig. 6(d). In order to satisfy the DOF quantity of ankle and reduce the structural complexity, a spherical bearing is arranged at ankle joint, which has three degrees of freedom to meet the needs of human motions. There is no angular sensor at the ankle joint, which makes the structure complex. However, the angle of ankle joint is necessary when calculating the torques required by the exoskeleton. In this study, it can be calculated by combining the angle of hip, knee and torso. The segment between ankle and foot transfers the exoskeleton's weight to the ground. The main structure of the foot is 3D-printed under the consideration of wear resistance and comfort. Inside the foot model, there are four force sensors to detect how much weight the human places on their left leg versus their right leg which is used by the control algorithm. The positions of the force sensors are considered which aims to acquire the contact force between the wearer and the ground in different condition of human motions. Notice that only the weight of the human is transferred onto the foot model and measured by the sensors. The wearer's boot rigidly attaches to the top of the exoskeleton's foot via a quick release binding.

\subsection{Structure and finite element analysis of the torque sensor}

The structure of the torque sensor is shown in Fig. 7, including inner base, outer base, supporting beam and strain beam. The inner base is connected with the joint axis, and the torque is transferred by a key. The outer base is connected with the joint connecter, and the torque is transferred by screw connections. Torque sensor and joint connecter are supported by joint axis, and there is almost no relative motion along the radial direction between them. The advantage of this design is that the influence of radial force on the stress distribution of the sensor can be reduced. Because of the limitation of the space inside the joint, the strain beam of the sensor was arranged along the axis instead of the radial direction. As shown in Fig. 7, the structure of double cantilever beam was designed on the strain beam. When applying moment on the torque sensor, the double cantilever beam structure deforms with the same deformation at diagonal position and opposite deformation at adjacent position. Strain gauges sticking at the both sides of the strain beam constitute a full bridge to convert physical deformation of the beam into voltage signal.

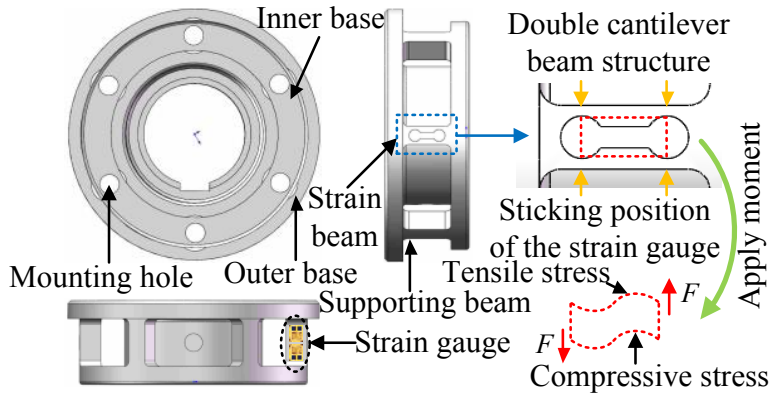

Figure 7. Structure and detection principle of the torque sensor For evaluating the performance of the torque sensor, finite element analysis was carried out using ANSYS workbench. Cylindrical constraint was applied to the axis hole, fixed constraint was applied to the mounting holes and moment was applied to the axis hole which is the same to the actual situation. After configuring material and meshing appropriately for the torque sensor and the process of calculation, the results of stress and strain distribution were acquired as shown in Fig. 8. It can be seen that the stress and strain at sticking positions are sensitive to the moment applied to the torque sensor because of the design of double cantilever beam structure.

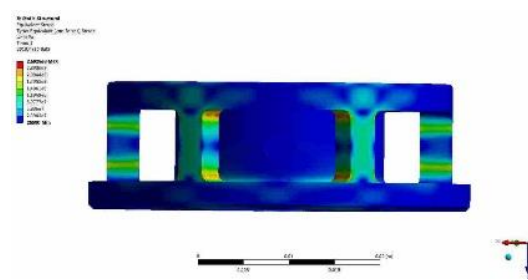

(a) Stress nephogram

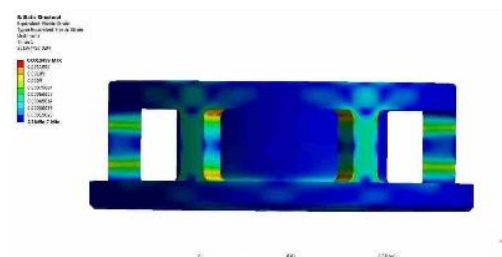

(b) Strain nephogram

Figure 8. The results of finite element analysis

\section{Design of controlling and sensing system}

When controlling the exoskeleton, actuating torques are calculated by dynamics model in real time which demands huge computation. Therefore, controller with high calculating speed and communication protocol with sufficient efficiency and reliability are desired.

The proposed controlling and sensing system is shown in Fig. 9. A stacked PC104 is adopted as the controller, which is connected to a computer to constitute a real-time simulation system with master-slave structure using xPC-Target toolbox of MATLAB. The system works based on CANopen communication protocol with advantages of high efficiency and reliability and being easy to extend. Two CAN buses are comprised in the exoskeleton to acquire the signal from sensors of right 
and left leg respectively. An inclinometer which mounts to the rear side of the back provides the absolute angle reference for control algorithm. Custom electronic boards are embedded into the joints to acquire joint angles and communicate with the controller. It is designed based on Hall Effect, which makes it possible to measure the relative angle precisely between two non-contact objects. Torque sensors detect the moments at hip and knee which can be used to obtain the interaction force between thighs and shanks of the exoskeleton and human legs during the movement. Four force sensors in each foot model form a detection system to acquire how much weight the human places on right and left leg respectively. The contact force information is used to determine the locomotion state of human-robot coupling system.

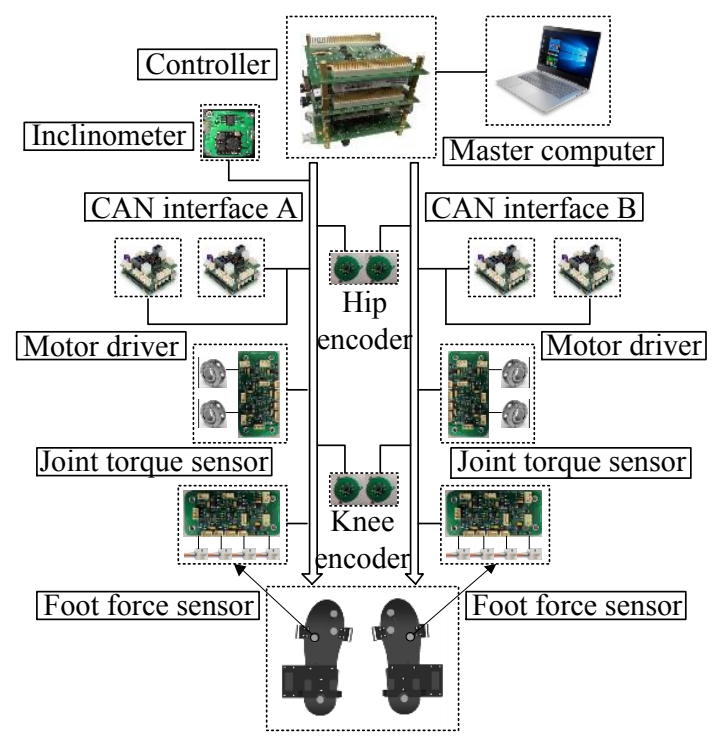

Figure 9. The controlling and sensing system

\section{Conclusions}

In this paper, a lightweight lower extremity exoskeleton is introduced. Approximating the exoskeleton kinematics and dynamics as the same as that of human leg allows the exoskeleton actuation to be designed based on biomechanics simulations of human body. This resulted in an exoskeleton leg with 7 DOF and two of them are actuated. To determine the parameters of the actuators, biomechanics simulations of human body of walking on flat ground, ascending stairs and sitting down and standing up are carried out. The weight of the exoskeleton is significantly reduced due to the use of carbon fiber material and compact structural design. For the sake of control, a torque sensor is designed and installed inside the joint of the exoskeleton which constitutes the sensing system with other sensors like angular sensors, force sensors and inclinometer to acquire motion data of the wearer. Additionally, finite element analysis of the torque sensor was carried out.

While there is still significant work remaining, the mechanical structure and electronic control system have been accomplished. Our future work will focus on the control strategy of the exoskeleton which includes achieving feed forward control to support the weight of the wearer in addition to supporting its own weight and integrating balance control to help maintain stability during the movement. In this way, the exoskeleton will have the potential to put the wearer in a slight weightless state, thus reducing power consumption. We anticipate eventual deployment of this exoskeleton as a wearable auxiliary and rehabilitation training device outside of the lab.

\section{Acknowledgment}

This work was supported by the National Key R\&D Program of China (Grant 2017YFB1302301), and the Joint Research Fund (U1713201\&U1613219) between the National Nature Science Foundation of China (NSFC) and Shen Zhen.

\section{References}

1. S. Goemaere, M. Van Laere, P. De Neve, and J. Kaufman, "Bone mineral status in paraplegic patients who do or do not perform standing.," Osteoporos Int., vol. 4, pp. 138-43, 1994.

2. Zeilig G, Weingarden H, Zwecker M, Dudkiewicz I, Bloch A, Esquenazi A. Safety and tolerance of the ReWalk exoskeleton suit for ambulation by people with complete spinal cord injury: a pilot study[J]. J Spinal Cord Med. 2012, 35(2): 96-101.

3. Strickland E. Good-bye, wheelchair[J]. Spectrum, IEEE, 2012, 49(1): 30-32.

4. Sankai Y. Leading edge of cybernics: Robot suit hal[C]//SICE-ICASE, 2006. International Joint Conference. IEEE, 2006: P-1-P-2.

5. A Tsukahara, Y Hasegawa, Y Sankai. Standing-up motion support for paraplegic patient with Robot Suit HAL[J]. 2009 IEEE International Conference on Rehabilitation Robotics, 2009: 211-217.

6. R.J. Farris, H.A. Quintero, and M. Goldfarb. Preliminary Evaluation of a Powered Lower Limb Orthosis to Aid Walking in Paraplegic Individuals[J]. Neural Systems and Rehabilitation Engineering, IEEE Transactions on, 2011, 19(6): 652-659.

7. Farris R J, Quintero H A, Murray S A, et al. A preliminary assessment of legged mobility provided by a lower limb exoskeleton for persons with paraplegia[J]. Neural Systems and Rehabilitation Engineering, IEEE Transactions on, 2014, 22(3): 482-490.

8. Chen C, Zheng D, Peng A, et al. Flexible design of a wearable lower limb exoskeleton robot[C]/Robotics and Biomimetics (ROBIO), 2013 IEEE International Conference on. IEEE, 2013: 209-214.

9. Huang $\mathrm{R}$, Cheng $\mathrm{H}$, Chen $\mathrm{Q}$, et al. Interactive learning for sensitivity factors of a human-powered augmentation lower exoskeleton[C]// Intelligent Robots and Systems (IROS), 2015 IEEE/RSJ International Conference on. IEEE, 2015: 6409-6415. 